\title{
Systemic oxidative stress in congenital central hypoventilation syndrome
}

\author{
To the Editor:
}

Congenital central hypoventilation syndrome (CCHS), also known as Ondine's curse, is a rare disorder characterised by severe hypoventilation during sleep and autonomic dysregulation $[1,2]$. The incidence of CCHS is about one in 200000 live births. In $>90 \%$ of cases, polyalanine repeat expansion mutations are present in the paired-like homeobox $P H O X 2 B$ gene, although a frameshift variant may also be found [3]. The $P H O X 2 B$ gene encodes a highly conserved homeobox domain transcription factor that plays a regulatory role in the differentiation of the motor neuron and the serotonergic neuronal fate in the development of the central nervous system [4]. The hallmark of CCHS is the "forgotten breathing", which implies the need for life-long mechanical ventilation during sleep. However, in more severe cases characterised by global hypoventilation, mechanical ventilation must also be extended during wakefulness. Ventilatory support may be provided by tracheostomy and assisted ventilation, noninvasive ventilation or diaphragm pacemakers. Severe respiratory depression typically arises at birth but in milder cases, CCHS may be diagnosed later on in childhood or adulthood (later-onset CCHS) $[1,2]$. The clue to the respiratory defect is a reduced response to hypercapnia and hypoxaemia depending on the malfunctioning of brainstem areas such as the retrotrapezoid nucleus, the parafacial respiratory group and the pre-Bötzinger complex, which are involved in the chemosensory drive to breathe [5-7]. Due to the key role played by $P H O X 2 B$ in the development of the autonomic nervous system, this genetic defect may also have several pathological consequences such as Hirschsprung's disease, neural crest tumours like neuroblastomas, decreased heart-rate variability, attenuated heart-rate response to exercise, constipation, oesophageal dysphagia, ophthalmological abnormalities, altered perception of anxiety, sporadic profuse sweating and hypothermia [1, 2]. Deficiencies in neurocognitive performance in preschool and school-aged children with CCHS have also been reported [8]. Although the brain injury found in CCHS patients appears to progress in several selected areas [9], the mechanisms underlying these abnormalities remain elusive. Unless CCHS is promptly diagnosed, brain lesions may be caused by intermittent hypoxia due to primary breathing impairment in early postnatal life. However, chronic episodes of hypoxiareoxygenation can occur during both nocturnal assisted ventilation and wakefulness when CCHS patients are engaged in activities like studying or relaxing (watching television, playing computer games, etc.). Under these conditions, the overproduction of reactive oxygen species (ROS) may provoke oxidative stress capable of inducing severe cellular damage to lipids, proteins and DNA, and activating apoptotic signalling [10]. Oxidative stress is closely associated with several chronic and acute disorders including atherosclerosis, neurodegenerative diseases (e.g. Alzheimer's and Parkinson's), cancer, diabetes mellitus and inflammatory diseases, as well as ageing process [11]. Interestingly, in breathing disorders characterised by intermittent hypoxia such as obstructive sleep apnoea syndrome, sudden infant death syndrome and Rett syndrome, an increased production of ROS has already been reported [12-14]. The aim of this study was to investigate the level of oxidative stress by measuring the intracellular ROS production in clinically stable CCHS patients. We analysed the systemic redox status in 14 patients with $P H O X 2 B$ mutation-confirmed CCHS and 14 healthy controls randomly selected from a cohort of healthy sex- and age-matched subjects who had been assessed for the absence of previous diseases possibly affecting the redox status, had a healthy lifestyle and were not taking drugs.

This study was approved by our institutional review board. Written informed consent/assent was obtained from all patients.

@ERSpublications

This study is the first to demonstrate an increase in ROS in CCHS patients. This implies that therapeutic strategies based on antioxidants should be taken in consideration and the mechanisms provoking the increase of ROS should be clarified. http://ow.ly/5Rhp30lJ7Yi

Cite this article as: Degl'Innocenti D, Becatti M, Peruzzi M, et al. Systemic oxidative stress in congenital central hypoventilation syndrome. Eur Respir J 2018; 52: 1801497 [https://doi.org/10.1183/13993003.014972018]. 
The demographic and clinical characteristics of the CCHS patients are reported in figure 1a. All patients in the study were only ventilated during sleep and none of them were suffering from pulmonary hypertension.

Systemic oxidative stress was analysed in the CCHS patients by evaluating intracellular ROS production in the leukocyte and erythrocyte populations. As previously reported [15], peripheral leukocytes which can be considered a "time-persistent" system reflecting the condition of the whole organism and therefore represent a valuable model for studying systemic oxidative stress-related disorders. Conversely, erythrocytes are particularly exposed to oxidative stress during their lifetime due to the high content of polyunsaturated fatty acids in their membranes and auto-oxidation of haemoglobin inside the cell [16]. In order to rule out any possible interference by recent episodes of hypoxia, the patients recruited underwent a complete full-night polysomnography and capnography, and their apnoea-hypopnoea index, oxygen desaturation index and carbon dioxide levels were all within the normal ranges. Fasting blood samples were collected from the antecubital vein using EDTA as an anticoagulant between 08:00 and 09:00 h. Samples were

\begin{tabular}{cccccc} 
a) & \multicolumn{1}{c}{} & \\
\hline Patient & PHOX2B mutation & Age years & Sex & Disease & Ventilation \\
\hline 1 & PolyAla 20/25 & 3 & M & CCHS & NIV \\
2 & PolyAla 20/25 & 5 & M & CCHS & NIV \\
3 & PolyAla 20/25 & 24 & F & CCHS & NIV \\
4 & PolyAla 20/25 & 28 & F & CCHS & Diaphragm pacing \\
5 & PolyAla 20/25 & 40 & F & CCHS & NIV \\
6 & PolyAla 20/26 & 7 & F & CCHS & Tracheostomy \\
7 & PolyAla 20/26 & 9 & M & CCHS + HSCR & Tracheostomy \\
8 & PolyAla 20/26 & 23 & M & CCHS & Diaphragm pacing \\
9 & PolyAla 20/26 & 25 & F & CCHS & Diaphragm pacing \\
10 & PolyAla 20/26 & 29 & M & CCHS & NIV \\
11 & PolyAla 20/27 & 5 & F & CCHS & Tracheostomy \\
12 & PolyAla 20/33 & 18 & F & CCHS & Diaphragm pacing \\
13 & FS c.722_759 & 4 & M & CCHS + HSCR & NIV \\
14 & FS c.930insG & 13 & F & CCHS + HSCR + NB & Tracheostomy \\
\hline
\end{tabular}
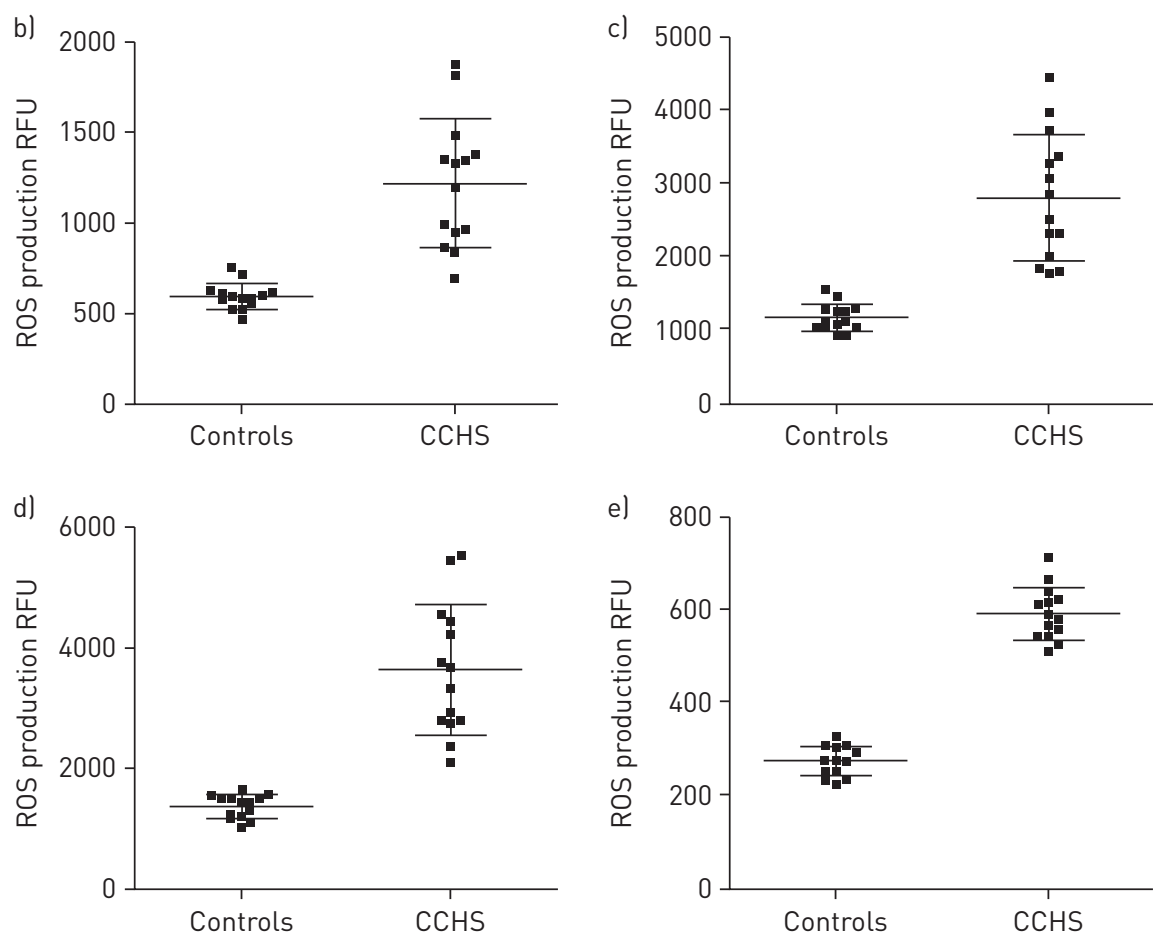

FIGURE 1 a) Summary of the characteristics of congenital central hypoventilation syndrome (CCHS) patients and the production of intracellular reactive oxygen species (ROS) in CCHS patient and control b) lymphocytes, c) monocytes, d) granulocytes and el erythrocytes. PolyAla: polyalanine; FS: frameshift; M: male; F: female; HSCR: Hirschprung's disease; NB: neuroblastoma; NIV: noninvasive ventilation; RFU: relative fluorescence unit. 
processed within $2 \mathrm{~h}$ after collection. The leukocytes were separated from the whole blood using BD FACS Lysing Solution (BD Biosciences, Franklin Lakes, NJ, USA). After collection, EDTA-anticoagulated blood $(100 \mu \mathrm{L})$ was resuspended in $2 \mathrm{~mL}$ of BD FACS Lysing Solution (BD Biosciences), mixed gently and incubated at room temperature in the dark for $10 \mathrm{~min}$. After being centrifuged, the supernatant was discarded and the cells washed twice with PBS. To determine the level of intracellular ROS, the cells were incubated with $2^{\prime}, 7^{\prime}$-dichlorodihydrofluorescein diacetate $(2.5 \mu \mathrm{M})$ (Invitrogen, Carlsbad, CA, USA) in RPMI medium without either serum or phenol red for $15 \mathrm{~min}$ at $37^{\circ} \mathrm{C}$. After labelling, the cells were washed, resuspended in PBS and immediately analysed using a FACSCanto flow cytometer (BD Biosciences). Cell viability, controlled by flow cytometry with propidium iodide staining, was found to exceed 95\%. The erythrocyte ROS production was analysed as previously reported [16]. For a single analysis, the fluorescence signals of 100000 erythrocytes were collected. Data were analysed using BD FACSDiva software (BD Biosciences) and the differences between sample groups were evaluated statistically using the unpaired t-test. As illustrated in figure 1b, the leukocyte ROS production in CCHS patients increased significantly in the subpopulation analysed compared with the healthy controls (lymphocyte ROS: $1219 \pm 356$ versus $595 \pm 73$ relative fluorescence units (RFU), p<0.0001, Cohen's d 1.54; monocyte ROS: $2795 \pm 868$ versus $1145 \pm 189$ RFU, $\mathrm{p}<0.0001$, Cohen's d 1.67; granulocyte ROS: $3641 \pm 1087$ versus $1365 \pm 196 \mathrm{RFU}, \mathrm{p}<0.0001$, Cohen's $\mathrm{d} 1.83$ ). These results were confirmed by the erythrocyte ROS production analysis, where a significant increase in CCHS erythrocyte ROS was evident (590 \pm 58 versus $270 \pm 31, \mathrm{p}<0.0001$, Cohen's d 3.23). Interestingly, our results are in agreement with those obtained in blood cells and sera of patients diagnosed with obstructive sleep apnoea, although different oxidative stress markers were assessed with other methods [17].

Conversely, we found no association between the ROS levels and other patient features, including age, sex, type of mutation and type of ventilator support. Chronic episodes of hypoxia-reoxygenation due to ventilatory impairment, which may occur during nocturnal assisted ventilation, as well as hypoxaemic episodes during wakefulness, may play a key role in ROS generation even though the ultimate triggers that cause the redox imbalance are unknown. ROS overproduction might cause damage to important biomolecules and organs with a plausible impact on the whole organism.

It is important to stress that the relevant advances in CCHS management in childhood have led to an increased survival rate, which implies that adult patients may now have to cope with an imbalanced ageing process. In conclusion, this study provides first-line evidence of the systemic oxidative status in CCHS patients, demonstrating an increase in oxidative stress in this rare disease. Many aspects must still be clarified, mostly related to the mechanisms provoking the increase of ROS; however, therapeutic strategies based on antioxidants, as well as the promotion of a healthy lifestyle (i.e. adequate physical exercise and a balanced diet), must be taken into consideration in order to mitigate the oxidative derangement in these patients.

Donatella Degl'Innocenti ${ }^{1,4}$, Matteo Becatti ${ }^{1,4}$, Marta Peruzzi $^{2}$, Claudia Fiorillo ${ }^{1}$, Matteo Ramazzotti ${ }^{1}$, Niccolò Nassi ${ }^{2}$, Cinzia Arzilli ${ }^{3}$ and Raffaele Piumelli ${ }^{2}$

${ }^{1}$ Dept of Experimental and Clinical Biomedical Sciences "Mario Serio", University of Florence, Florence, Italy. ${ }^{2}$ Sleep Breathing Disorders and SIDS Center, Meyer Children's Hospital, Florence, Italy. ${ }^{3}$ Dept of Neuroscience, Psychology, Drug Research and Child Health, University of Florence, Florence, Italy. ${ }^{4}$ These authors contributed equally.

Correspondence: Raffaele Piumelli, Sleep Breathing Disorders and SIDS Center, Meyer Children's Hospital, Viale Pieraccini 24, 50132 Florence, Italy. E-mail: raffaele.piumelli@meyer.it

Received: June 122018 | Accepted after revision: Aug 292018

Acknowledgements: The authors are grateful to the Associazione Italiana per la Sindrome da Ipoventilazione Centrale Congenita (A.I.S.I.C.C.) and to all the CCHS patients and their families for having allowed the enrolment of their children in this work.

Conflict of interest: None declared.

Support statement: This work was supported by a grant from the University of Florence (Fondi di Ateneo 2017 to D. Degl'Innocenti).

\section{References}

1 Weese-Mayer DE, Berry-Kravis EM, Ceccherini I, et al. An official ATS clinical policy statement: congenital central hypoventilation syndrome: genetic basis, diagnosis, and management. Am J Respir Crit Care Med 2010; 181: 626-644.

2 Weese-Mayer DE, Rand CM, Zhou A, et al. Congenital central hypoventilation syndrome: a bedside-to-bench success story for advancing early diagnosis and treatment and improved survival and quality of life. Pediatr Res 2017; 81: 192-201.

3 Amiel J, Laudier B, Attié-Bitach T, et al. Polyalanine expansion and frameshift mutations of the paired-like homeobox gene PHOX2B in congenital central hypoventilation syndrome. Nat Genet 2003; 33: 459-461. 
4 Pattyn A, Simplicio N, van Doorninck JH, et al. Ascl1/Mash1 is required for the development of central serotonergic neurons. Nat Neurosci 2004; 7: 589-595.

5 Champagnat J, Morin-Surun MP, Bouvier J, et al. Prenatal development of central rhythm generation. Respir Physiol Neurobiol 2011; 178: 146-155.

6 Dubreuil V, Ramanantsoa N, Trochet $\mathrm{D}$, et al. A human mutation in $\mathrm{PHOX}_{2} \mathrm{~B}$ causes lack of $\mathrm{CO}_{2}$ chemosensitivity, fatal central apnea, and specific loss of parafacial neurons. Proc Natl Acad Sci USA 2008; 105: 1067-1072.

7 Perez IA, Keens TG. Peripheral chemoreceptors in congenital central hypoventilation syndrome. Resp Physiol Nerobiol 2013; 185: 186-193.

8 Zelko FA, Stewart TM, Brogadir CD, et al. Congenital central hypoventilation syndrome: Broader cognitive deficits revealed by parent controls. Pediatr Pulmonol 2018; 53: 492-497.

9 Kumar R, Woo MS, Macey PM, et al. Progressive gray matter changes in patients with congenital central hypoventilation syndrome. Pediatr Res 2012; 71: 701-706.

10 Rahal A, Kumar A, Singh V, et al. Oxidative stress, prooxidants, and antioxidants: the interplay. Biomed Res Int 2014; 2014: 761264.

11 Poprac P, Jomova K, Simunkova M, et al. Targeting free radicals in oxidative stress-related human diseases. Trends Pharmacol Sci 2017; 38: 592-607.

12 Lavie L. Oxidative stress in obstructive sleep apnea and intermittent hypoxia - revisited - The bad ugly and good: implications to the heart and brain. Sleep Med Rev 2015; 20: 27-45.

13 Prandota J. Possible pathomechanisms of sudden infant death syndrome: key role of chronic hypoxia, infection/ inflammation states, cytokine irregularities, and metabolic trauma in genetically predisposed infants. Am J Ther 2004; 11: 517-546

14 Bebensee DF, Can K, Müller M. Increased mitochondrial mass and cytosolic redox imbalance in hippocampal astrocytes of a mouse model of rett syndrome: subcellular changes revealed by ratiometric imaging of JC-1 and roGFP1 fluorescence. Oxid Med Cell Longev 2017; 2017: 3064016.

15 Becatti M, Emmi G, Silvestri E, et al. Neutrophil activation promotes fibrinogen oxidation and thrombus formation in Behçet disease. Circulation 2016; 133: 302-311.

16 Becatti M, Marcucci R, Gori AM, et al. Erythrocyte oxidative stress is associated with cell deformability in patients with retinal vein occlusion. J Thromb Haemost 2016; 14: 2287-2297.

17 Eisele HJ, Markart P, Schulz R. Obstructive sleep apnea, oxidative stress, and cardiovascular disease: evidence from human studies. Oxid Med Cell Longev 2015; 2015: 608438. 